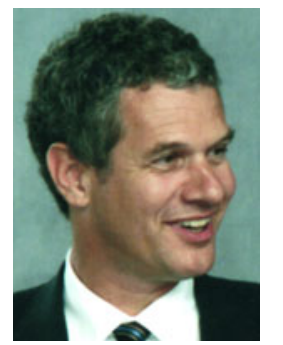

Matthias R. Lemke

\title{
Psychotische Störungen bei Patienten mit Morbus Parkinson
}

Matthias R. Lemke

Rheinische Kliniken Bonn

psychoneuro 2005; 31 (9): 417-421

$\mathrm{P}$ sychosen treten bei $10-40 \%$ der Patienten mit Morbus Parkinson auf. Bei medikamentös unbehandelten Patienten sind sie seltener und manifestieren sich meist im Zusammenhang mit der dopaminerg wirksamen Antiparkinsonmedikation (14). Die Entwicklung einer Psychose bei Patienten mit Morbus Parkinson verstärkt die Behinderung durch die Erkrankung und ist nicht selten der Grund für eine stationäre Krankenhausbehandlung oder Heimunterbringung der Patienten (9).

Die zu verzeichnende steigende Prävalenz von Psychosen bei Morbus Parkinson ist zum Teil auf die erhöhte Lebenserwartung der Patienten, zum Teil auch als Folge der immer erfolgreicheren Therapie der motorischen Symptome des Morbus Parkinson zurückzuführen. Die Symptome der Psychose bei Morbus Parkinson zeigen ein ebenso vielgestaltiges Spektrum wie die anderen nicht-motorischen und motorischen Symptome der Erkrankung. Psychosen manifestieren sich bei den Patienten als Störungen der Wahrnehmung, vor allem als optische Halluzinationen, des inhaltlichen Denkens als Wahnerleben, als dysphorische Stimmung, Antriebssteigerung und gesteigertes, enthemmtes Sexualverhalten.

Psychosen treten bei 10-40\% der Patienten mit Morbus Parkinson auf. Als erste Maßnahme sollten mögliche infektiöse, toxische oder metabolische Ursachen, insbesondere Hydratationsund Elektrolytstörungen, wenn notwendig, behandelt werden. Anti-Parkinson-Medikamente wie Anticholinergika, Selegelin, Dopaminagonisten und COMT-Inhibitoren sollten reduziert oder wenn möglich abgesetzt werden. Die Dosis von Levodopa sollte soweit wie möglich reduziert werden, wobei sich die motorischen Symptome in der Regel verschlechtern werden. Daher wird oft, ebenso wie in krisenhaften Zuspitzungen der Symptome, eine neuroleptische Medikation notwendig sein, wobei wegen ihres geringeren Risikos, extrapyramidale motorische Symptome zu induzieren oder zu verstärken, neuere Neuroleptika indiziert sind. Der Einsatz von Risperidon und Olanzapin wird wegen möglicher extrapyramidaler motorischer Effekte bei Patienten mit Morbus Parkinson nicht empfohlen. Die meisten Daten, auch kontrollierter Studien, zeigen eine gute Wirksamkeit für Clozapin, wobei Sedierung, Speichelfluss und Störungen der Orthostase sowie die Gefahr der Agranulozytose den Einsatz limitieren. Alternativ stehen Quetiapin und Ziprasidon zur Verfügung. Für Quetiapin liefern offene Studien und Kasuistiken an mehr als 200 Patienten mit Morbus Parkinson Hinweise auf gute Wirksamkeit und Verträglichkeit. Für Ziprasidon liegen Daten offener Studien vor, wobei hier auch die parenterale Gabe gute Wirksamkeit und Verträglichkeit in der Akutbehandlung bei agitierten Patienten mit Morbus Parkinson zeigte. Keine Daten liegen zu Amisulprid und Aripiprazol vor.

\section{Pathophysiologie}

Die motorischen Symptome des Morbus Parkinson sind auf ein Dopamindefizit als Folge degenerativer Veränderung dopaminerger Neurone vor allem im Mittelhirn und Striatum zurückzuführen (7). Mit fortschreitender Erkrankung kommt es zu einer postsynaptischen Hypersensitivität der Dopaminrezeptoren, die auch für die Levodopa induzierten Dyskinesien und Fluktuationen verantwortlich gemacht werden (11).

Frontaler Kortex, limbisches System und Projektionen zu den Basalganglien sind beteiligt an der Regu- lation von Emotionen und Entstehung von Psychosen. Neuere Untersuchungen zeigen, dass die degenerativen Veränderungen beim Morbus Parkinson nicht auf die Basalganglien beschränkt sind, sondern auch andere Strukturen, wie z.B. die limbische Schleife, betreffen (1). Postsynaptische Hypersensitivität monoaminerger Rezeptoren in genau diesen Strukturen des zentralen Nervensystems könnte daher analog zu der bekannten Hypersensitivität dopaminerger Rezeptoren in den motorischen Schleifen das Entstehen psychotischer Symptome bedin- 


$\begin{array}{cl}\text { Tab. } 1 & \text { Risikofaktoren für das } \\ & \text { Auftreten psychotischer } \\ & \text { Symptome bei Morbus } \\ & \text { Parkinson }\end{array}$

- Höheres Lebensalter

- Fortgeschrittenes Krankheitsstadium

- Schlafstörungen

- Demenz

- Somatische Komorbidität

- Polypharmazie

\section{Tab. 2 Vorboten für die Mani- festation einer Psychose bei Patienten mit Morbus Parkinson}

- Fortschreitende kognitive Defizite

- Schlafstörungen

- Persönlichkeitsveränderungen

- Hypersexualität

- Optische Halluzinationen

- Wahnhafte Gedanken

- Agitation, Aggressivität

gen (11). Eine Hypersensitivität serotonerger Rezeptoren als Folge der Degeneration serotonerger Projektionen vom Nucleus raphe des Hirnstammes zu kortikalen Arealen könnte ebenfalls ein Grund für die Manifestation psychotischer Störungen bei Patienten mit Morbus Parkinson sein. Dies könnte auch die besondere antipsychotische Wirksamkeit neuerer Neuroleptika erklären, die eine Affinität für dopaminerge und serotonerge Rezeptoren aufweisen.

Untersuchungen zum Zusammenhang zwischen Schlafstörungen und Psychose deuten darauf hin, dass es speziell einen Zusammenhang zwischen bestimmten Störungen des REM-Schlafes und dem Auftreten von Halluzinationen geben könnte. Der Zusammenhang zwischen kognitiven Einschränkungen und Psychose bei Patienten mit Morbus Parkinson gab Anlass zu der Hypothese, dass auch andere als die monoaminergen Neurotransmitter an der Entstehung der Psychose beteiligt sein könnten, was entsprechende therapeutische Konsequenzen hätte (s.u.) (11).

\section{Risikofaktoren und klinisches Bild}

Es sind eine Reihe von Risikofaktoren bekannt, die die Wahrscheinlichkeit für die Manifestation psychotischer
Symptome bei Patienten mit Morbus Parkinson erhöhen (Tab. 1). In der Regel treten die Symptome der Psychose nicht abrupt in voller Ausprägung auf, sondern entwickeln sich langsam. Dieser Prozess ist progredient und durch bestimmte Vorboten und Frühsymptome charakterisiert (Tab. 2).

Bei nicht dementen Patienten mit Morbus Parkinson entwickeln sich die Symptome der Psychose in der Regel schrittweise über eine leichte Beeinträchtigung kognitiver Defizite, Schlafstörungen, Persönlichkeits- und Verhaltensänderungen, Störungen der Wahrnehmung und des inhaltlichen Denkens (Wahn). Schlafstörungen manifestieren sich als lebhafte Träume oder Alpträume, Reduktion der Schlafqualität, Ein- und Durchschlafstörungen, Reduktion der Gesamtschlafzeit, Tagesmüdigkeit, motorische Aktivität in REM-Phasen (REM-sleep behavior disorder) und nächtliche Myokloni. Eine prospektive Untersuchung über sechs Jahre konnte den Zusammenhang zwischen Schlafstörungen und Halluzinationen nicht bestätigen, wobei die Untersuchungsintervalle relativ lang waren (4). Diese Studie belegt allerdings auch die hohe Inzidenz (62\%) von Halluzinationen bei Patienten mit Morbus Parkinson.

Hinsichtlich der Persönlichkeit stellen sich oft subtile Veränderungen ein. Die betroffenen Patienten werden fordernder gegenüber ihren Angehörigen, werden gereizter, zeigen eine geringere Toleranz gegenüber Frustrationen, werden egozentrischer und verkennen ihre eigenen Einschränkungen und die Belastung für die sie versorgenden Menschen. Dieses Verhalten stellt einen deutlichen Gegensatz zu Ausprägungen prämorbider Persönlichkeitsstrukturen wie Ordentlichkeit, Pünktlichkeit, Reserviertheit und Kontrolliertheit dar. Bei weiter erhaltener Einsichtsfähigkeit entwickeln sich dann optische Halluzinationen, die aus illusionären Verkennungen z.B. von Lichtschatten entstehen. Akustische oder taktile Halluzinationen sind extrem selten. Anfangs mögen diese Halluzinationen hauptsächlich in der Dämmerung, später dann auch tagsüber auftreten. Bei den Wahninhalten dominieren Verfolgungs- und Beeinträchtigungswahn, Misstrauen und häufig Eifersuchtswahn. Bei weiterer Progredienz der Psychose treten Agitation und aggressives Verhalten hinzu,
Zyprexa $^{\oplus}$ Filmtabletten: Zyprexa $2,5 \mathrm{mg}$, Zyprexa $5 \mathrm{mg}$, Zyprexa ${ }^{\oplus} 7,5 \mathrm{mg}$, Zyprexa

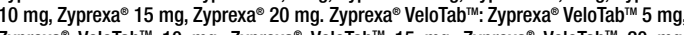
Zyprexa $^{\oplus}$ VeloTab $^{T M} 10 \mathrm{mg}$, Zyprexa ${ }^{\oplus}$ VeloTab $^{T M} 15 \mathrm{mg}$, Zyprexa ${ }^{\oplus}$ VeloTab $^{\text {TM }} 20 \mathrm{mg}$, Zyprexa $^{\circledast} 10 \mathrm{mg}$ Pulver zur Herstellung einer Injektionslösung. Wirkstoff: Olanzapin. Zusammensetzung: 1 Filmtablette enthält $2,5 \mathrm{mg}, 5 \mathrm{mg}, 7,5 \mathrm{mg}, 10 \mathrm{mg}, 15 \mathrm{mg}$ oder $20 \mathrm{mg}$ Olanzapin. 1 Zyprexa VeloTab $^{\text {TM }}$ Schmelztablette enthält $5 \mathrm{mg}, 10 \mathrm{mg}, 15 \mathrm{mg}$ oder $20 \mathrm{mg}$ Olanzapin. Zyprexa $10 \mathrm{mg}$ Pulver zur Herstellung einer Injektionslösung: $10 \mathrm{mg}$ Olanzapin je propylcellulose . Weite Bestandteile: Filmtabletten: Laktose-Monohydrat, HydroxyKarnaubawachs. 2,5 mg, 5 mg, 7,5 mg und 10 mg Filmtabletten: essbare blaue Tinte, Farbmischung weiB (Hypromellose, Titandioxid E 171. Macrogol, Polysorbat 80). $15 \mathrm{mg}$ Filmtabletten: Farbmischung hellblau (Titandioxid E 171, Laktose-Monohydrat, Hypromellose, Tritabletten: Farbmischung hellblau (Titandioxid E 171, Laktose-Monohydrat, Hypromellose, Tri-
acetin, Indigocarmin E 132). 20 mg Filmtabletten: Farbmischung rosa (Titandioxid E 171 , Macrogol, Laktose-Monohydrat, Hypromellose, synthetisches rotes Eisenoxid). VeloTab Schmelztabletten: Gelatine, Mannitol, Aspartam, woraus Phenylalanin gebildet wird, Natriummethyl-4-hydroxybenzoat, Natriumpropyl-4-hydroxybenzoat. Zyprexa ${ }^{\circledast}$ Pulver zur Herstellung einer Injektionslösung: Laktose-Monohydrat, Weinsäure E 334, Salzsäure, Natriumhydroxid. An-wendungsgebiete: Filmtabletten und VeloTab ${ }^{\top \mathrm{T}}$ : Schizophrenie. Bei Patienten, die initial auf die Behandlung angesprochen haben, ist Zyprexa ${ }^{\circledast}$ zur Aufrechterhaltung der klinischen Besserung wirksam. Mäßig schwere bis schwere manische Episoden. Bei Patienten, deren manische Episode auf eine Behandlung mit Olanzapin angesprochen hat, ist Olanzapin zur Phasenprophylaxe bei bipolaren Störungen angezeigt. Zyprexa ${ }^{\circledast}$ Pulver zur Herstellung einer Injektionslösung: schnelle Beherrschung von Agitation und gestörtem Verhalten bei Patienten mit Schizophrenie oder manischen Episoden, wenn eine orale Therapie nicht geeignet ist. Die Behandlung sollte sobald als möglich beendet und mit der oralen Anwendung von Olanzapin begonnen werden. Gegenanzeigen: Bekannte Überempfindlichkeit gegen Olanzapin oder einen der Hilfsstoffe; bekanntes Risiko eines Engwinkelglaukoms. Nebenwirkungen: Seh häufig: Schläfrigkeit, Gewichtszunahme. In klinischen Studien bei Patienten mit Demenzbedingter Psychose, höhere Inzidenz an Todesfällen und zerebrovaskulären Ereignissen sowie sehr häufig abnormer Gang und Stürze. Häufig: Verschlechterung der Parkinson-Symptome und Halluzinationen bei Parkinson'scher Erkrankung. Eosinophilie, Zunahme des Appetits, erhöhter Glukose- und Triglyceridspiegel, Schwindelgefühl, Akathisie, Parkinsonismus, Dyskinesie, orthostatische Hypotonie und leichte, vorübergehende anticholinerge Effekte. Besonders zu Behandlungsbeginn vorübergehende asymptomatische Erhöhungen der Lebertransaminasen. Asthenie, Ödeme. In einer klinischen Prüfung bei Patienten mit bipolarer Manie betrug bei einer gleichzeitigen Behandlung mit Valproat und Olanzapin die Häufigkeit einer Neutropenie 4,1\%. Bei älteren Patienten mit Demenz Pneumonie, erhöhte Körpertemperatur, Lethargie, Erythem, visuelle Halluzinationen und Harninkontinenz. Gelegentlich: Lichtüberempfindlichkeitsreaktionen, Bradykardie mit oder ohne Hypotonie oder Synkope, Sprachstörungen, hohe Kreatin-Phosphokinasewerte. Selten: Leukopenie, Ausschlag, Krampfanfälle, meistens mit Krampfanfällen in der Anamnese oder Risikofaktoren. Sehr selten: Thrombozytopenie, Neutropenie, allergische Reaktion (z.B. anaphylaktoide Reaktion, Angioödem, Juckreiz oder Nesselsucht), Hyperglykämie und/oder Entwicklung oder Verschlechterung eines Diabetes, gelegentlich begleitet von Ketoacidose oder Koma, einschließlich einiger fataler Fälle, Hypothermie. Malignes neuroleptisches Syndrom (MNS) Thromboembolien, einschl. Lungenembolien und tiefer Venenthrombose. Pankreatitis, Thromboembolien, einschl. Lungenembolien und tiefer Venenthrombose, Pankreatitis,
Hepatitis, Rhabdomyolyse, Priapismus, Schwierigkeiten beim Wasserlassen. Wenn Olanzapin plötzlich abgesetzt wurde, akute Symptome wie Schwitzen, Schlaflosigkeit, Zittern, Angst, Ubelkeit oder Erbrechen. Plasmaprolaktinspiegel gelegentlich erhöht, damit zusammenhängende klinische Befunde (Gynäkomastie, Galaktorrhö, Brustvergrößerung) jedoch selten. Prolaktinspiegel meist normalisiert, ohne dass Behandlung abgebrochen wurde. Zyprexa ${ }^{\circledR}$ Pulver zur Herstellung einer Injektionslösung zusätzlich: häufig: Orthostatische Hypotonie, Hypotonie, Unbehagen an der Injektionsstelle, Bradykardie mit oder ohne Hypotonie oder Synkope, gelegentlich Sinuspause, gelegentlich Hypoventilation. Sehr selten wurde ein zeitlicher Zusammenhang zwischen einer Behandlung mit i.m. Olanzapin und Hypotonie, Bradykardie, Atemdepression gleichzeitig Benzodiazepine und/oder andere Antipsychotika oder höhere Dosen als empfohlen erhielten. Warnhinweise und Vorsichtsmaßnahmen: Ärztliche Überwachung bei Patienten mit Diabetes und Risikofaktoren für Diabetesentwicklung ratsam. Bei Behandlungsende schrittweise Dosisreduktion erwägen. Olanzapin kann Schläfrigkeit und Schwindel verursachen, der Patient sollte vor dem Bedienen gefährlicher Maschinen und Autofahren gewarnt werden. Strenge Indikationsstellung in der Schwangerschaft. Anwendung zur Behandlung von durch Arzneimittel mit dopaminerger Wirkung ausgelösten Psychosen bei Patienten mit Parkinson'scher Erkrankung nicht empfohlen. Olanzapin ist für die Behandlung von Psychosen und/oder Verhaltensstörungen bei Patienten mit Demenz nicht zugelassen; die Behandlung in dieser speziellen Patientengruppe wird nicht empfohlen, da Mortalität und Risiko zerebrovaskulärer Zwischenfälle in klinischen Studien erhöht waren. Filmtabletten enthalten Laktose. kularer Zwischentalle in kinischen Studien erhoht waren. Filmtabletten enthalten Laktose.
Vorsicht bei Patienten mit erhöhten ALT- und/oder AST-Werten oder Anzeichen einer Leberfunktionseinschränkung. Bei Hepatitis-Diagnose Olanzapin-Behandlung beenden. Vorsicht bei Patienten mit niedrigen Leukozyten- und/oder Neutrophilenwerten, Knochenmarksdepression/-toxizität in der Anamnese, bei hypereosinophilen Zuständen oder myeloproliferativer Erkrankung. Es liegen nur wenige Daten zur gleichzeitigen Behandlung mit Lithium und Valproat und keine Daten zur gleichzeitigen Behandlung mit Carbamazepin vor. Vorsicht bei Patienten mit Prostatahypertrophie, paralytischem lleus, Krampfanfällen in der Anamnese oder die Krampfschwelle verändernden Zuständen. Vorsicht bei gleichzeitiger Einnahme von Arzneimitteln, von denen bekannt ist, dass sie die QTc-Strecke verlängern. Bei Anzeichen einer Spätdyskinesie Dosisreduktion oder Behandlungsabbruch erwägen. VeloTab ${ }^{\text {TM: }}$ allergische Reaktion durch Konservierungsmittel Natriummethyl-4-hydroxybenzoat, Natriumpropyl-4 hydroxybenzoat. Wirksamkeit von i.m. Olanzapin bei Agitation und gestörtem Verhalten aufgrund von anderen Erkrankungen als einer Schizophrenie oder manischen Episoden nicht nachgewiesen. i.m. Olanzapin darf bei Patienten mit instabilen Zuständen, wie akutem Myokardinfarkt, instabiler Angina pectoris, schwerer Hypotonie und/oder Bradykardie, Sick Sinus Syndrom oder im Anschluss an eine Herzoperation nicht gegeben werden. Die gleichzeitige Gabe von i.m. Olanzapin und einem parenteralen Benzodiazepin wird nicht empfohlen. Falls notwendig sollte das parenterale Benzodiazepin nicht früher als eine Stunde nach der i.m. Olanzapin Injektion gegeben werden. Unbedenklichkeit und Wirksamkeit von i.m. Olanzapin bei Patienten mit Alkohol- oder Arzneimittelvergiftung nicht untersucht. Dosierung, Art und Dauer der Anwendung: Einzelheiten entnehmen Sie bitte der Gebrauchs- und Fachinformation, deren aufmerksame Durchsicht wir empfehlen. Verschreibungspflichtig. Fachinformation, deren aufmerksame Durchsicht wir empfehlen. Verschreibungspflichtig. 5 mg: N2/28 Tbl., N3/56 Tbl.; Zyprexa ${ }^{\oplus} 7,5$ mg: N3/56 Tbl.; Zyprexa 10 mg: N2/28 Tbl., N3/56 Tbl.; Zyprexa ${ }^{\circledR} 15$ mg: N2/28 Tbl., N3/56 Tbl.; Zyprexa 20 mg: N2/28 Tbl.; Zyprexa VeloTab 5 mg: N2/28 Tbl., N3/56 Tbl.; Zyprexa ${ }^{\text {TM }}$ VeloTab ${ }^{\text {TM }} 10$ mg: N2/28 Tbl., N3/56 Tbl.; Zyprexa ${ }^{\circledast}$ VeloTab $^{\text {TM }} 15$ mg: N2/28 Tbl., N3/56 Tbl.; Zyprexa ${ }^{\circledast}$ VeloTab ${ }^{T M} 20$ mg: N2/28 Tbl.; Zyprexa $^{\circledR} 10 \mathrm{mg}$ Pulver zur Herstellung einer Injektionslösung: N1/1 Durchstechflasche. Krankenhauspackungen. Lilly Deutschland GmbH, Gießen. Stand: Februar 2005

Besuchen Sie uns: www.zyprexa.de 
was die Möglichkeit der Versorgung durch Angehörige im häuslichen Setting limitiert und zur Hospitalisierung der Betroffenen führt.

\section{Behandlung der Psychose bei Morbus Parkinson}

Als erster Schritt sollten mögliche komorbide metabolische, toxische oder infektiöse Ursachen abgeklärt und korrigiert werden, die nicht selten eine nicht beachtete Ursache der psychotischen Symptome darstellen. Insbesondere sollte eine Überprüfung des Wasser- und Elektrolythaushaltes vorgenommen werden, der häufig bei Patienten im höheren Lebensalter gestört ist.

In einem weiteren Schritt sollte eine Anpassung der Antiparkinsonmedikation vorgenommen werden. Hierzu sollten der Reihe nach vorsichtig folgende Substanzklassen abgesetzt werden: Anticholinergika, Amantadine, Selegelin. Ein Erfolg ist nach einigen Tagen zu erwarten, wobei sich meistens zuerst der Schlaf verbessert. Als nächstes sollten Dopaminagonisten und langwirksame Dopaminomimetika abgesetzt werden. Ein völliges Absetzen der gesamten dopaminergen Medikation, sog. „drug holidays“, ist kontraindiziert und kann zu lebensbedrohlichen Komplikationen führen. Beschrieben wurden Zustände, ähnlich dem malignen neuroleptischen Syndrom, mit Rhabdomyolyse, Fieber, Stupor, Koma und Tod $(12,14)$. Falls der Patient Antidepressiva erhält, sollte die Notwendigkeit dieser Therapie kritisch überprüft werden, da die Therapie mit Antidepressiva bei Patienten mit Morbus Parkinson mit dem Auftreten einer Psychose assoziiert sein kann (8).

Bei diesem Vorgehen respondieren hinsichtlich der Symptome der Psychose initial ca. 50\% der Patienten mit Morbus Parkinson bezüglich der psychotischen Symptome. Bei vielen kommt es jedoch zu einer Verschlechterung der motorischen Symptome, so dass die Einleitung einer neuroleptischen Therapie unumgänglich ist. Falls die Patienten wegen krisenhafter Zuspitzung von Halluzinationen oder wahnhaften Denkstörungen stationär behandelt werden müssen, ist eine schritt- weise Reduktion der Antiparkinsonmedikation nicht möglich und eine sofortige Gabe und schnelle Aufdosierung von Neuroleptika notwendig, die auf die Symptome der Psychose in der Regel besser wirken als Benzodiazepine.

\section{Spezifische antipsychotische Therapie}

Da die Dosis-Wirkungskurven antipsychotischer und extrapyramidaler Effekte bei traditionellen Neuroleptika eng beieinander liegen, sind diese wegen verstärkender Wirkung auf die motorischen Defizite bei Patienten mit Morbus Parkinson kontraindiziert (9). Patienten mit Morbus Parkinson sind aufgrund der degenerativen Vorschäden in den Basalganglien und nigrostriatalen Projektionsbahnen (1) besonders empfindlich für extrapyramidal-motorische Effekte der Neuroleptika. Es sollten daher nur Substanzen verwendet werden, bei denen extrapyramidal-motorische und antipsychotische Dosis-Wirkungskurven möglichst weit voneinander entfernt liegen. Derzeit stehen als neuere Neuroleptika Clozapin, Amisulprid, Risperidon, Olanzapin, Quetiapin, Ziprasidon und Aripiprazol für die Behandlung der Psychose zur Verfügung. Für Amisulprid und Aripiprazol liegen keine Daten vor. Die Gabe von Risperidon und Olanzapin wird wegen deutlicher negativer Effekte auf die motorischen Symptome bei Patienten mit Morbus Parkinson nicht empfohlen (3). Allerdings muss darauf hingewiesen werden, dass unter neueren Neuroleptika bei Patienten im fortgeschrittenen Alter die Motalität erhöht ist, weshalb die FDA eine „boxed warning“ verfügt hat. Dies könnte auch für ältere Neuroleptika zutreffen, wurde aber nicht untersucht.

\section{Clozapin}

Die meisten, auch kontrollierten Daten, existieren zu Clozapin, das wirksam und nur mit sehr geringen und vorübergehenden Effekten auf die Motorik eingesetzt werden kann. Die Anfangsdosis sollte zwischen 6,25 und $12,5 \mathrm{mg} /$ Tag liegen, die mittlere therapeutische Dosis liegt bei 25 bis 37,5 mg/Tag, und eine ma- ximale Dosis von $100 \mathrm{mg} /$ Tag sollte nicht überschritten werden (14). Günstig ist die Antitremorwirkung der Substanz. Die sedierende Komponente kann bei Gabe am Abend genutzt werden. Wichtig ist eine langsame Aufdosierung, da sonst Unverträglichkeiten zum Absetzen führen und das Risiko eines deliranten Zustandes stark erhöht wird. Unter der Behandlung mit Clozapin besteht dosisunabhängig das Risiko der Agranulozytose, was mit häufigen Kontrollen des Blutbildes verbunden ist. Clozapin ist nur als Medikament dritter Wahl bei Patienten mit schizophrenen Psychosen zugelassen und unterliegt einer kontrollierten Verschreibung (6). Störungen der Orthostase, Hypotension und Sialorrhoe sind häufige, dosisabhängige Komplikationen $(14,15)$.

\section{Quetiapin}

Das am zweitbesten untersuchte Neuroleptikum bei Patienten mit Morbus Parkinson und Psychose ist Quetiapin. Es existieren offene Studien, in denen bei mehr als 200 Patienten eine gute Wirksamkeit auf die Symptome der Psychose gezeigt werden konnte. Nach einer vorsichtigen Eindosierung mit maximal 25 $\mathrm{mg} /$ Tag scheint eine therapeutische Dosis von $75 \mathrm{mg} /$ Tag ausreichend wirksam zu sein (14). Komplikationen sind Sedierung und orthostatische Hypotension sowie leichte Effekte auf die Motorik, die geringfügig stärker zu sein scheinen als bei Clozapin, obwohl hier keine vergleichenden, randomisierten Studien vorliegen.

\section{Ziprasidon}

Über Wirksamkeit und Verträglichkeit von Ziprasidon bei dieser Indikation liegen erste Daten vor. Ziprasidon zeigt in der Behandlung von Patienten mit schizophrenen Psychosen gute Wirksamkeit und ein geringes Risiko für extrapyramidal-motorische Effekte und tardive Dyskinesien auch bei höheren Dosierungen, geringe Sedierung und keine anticholinergen Wirkungen (13). Es scheint, dass auch eine wirksame und sichere Therapie bei Patienten mit Psychose und Morbus Parkinson durchgeführt werden 
kann. Diese Indikation wurde bis jetzt an mehreren Fallserien untersucht, wobei in einer Arbeit fünf Patienten im Rahmen einer Akutbehandlung wegen Agitiertheit Ziprasidon parenteral erhielten $(5,10$, unveröffentlichte Ergebnisse). Eingesetzt wurde Ziprasidon in einer Dosierung von 20 bis $40 \mathrm{mg} / \mathrm{Tag}$. Diese Indikation sollte weiter überprüft werden.

\section{Azetylcholinesteraseinhibitoren}

Ob Azetylcholinesteraseinhibitoren neben therapeutischen Effekten bei Patienten mit Morbus Parkinson und Demenz auch Wirksamkeit hinsichtlich der Psychose zeigen, ist Gegenstand gegenwärtiger Untersuchungen, die auf der Hypothese einer gemeinsamen Ätiologie dieser Störungen beruhen. Offene Studien mit Rivastigmin und Donezepil geben Hinweise auf eine Reduktion der Psychose, ohne die motorischen Störungen zu verschlechtern (14).

\section{Ausblick}

Halluzinationen und wahnhaftes Denken als Symptome einer Psychose sind häufige Komplikationen bei Patienten mit Morbus Parkinson mit erheblichen Konsequenzen für die Versorgung der Patienten. Bei der Sichtung der Literatur zu dieser Thematik erscheint es jedoch, dass die Grundlagen der Terminologie nicht immer klar sind und die Begriffe Halluzination, Wahn, Psychose und Delir ungenau definiert und differenziert werden. Angaben zu anderen psychopathologischen Aspekten psychotischer Störungen wie z.B. Störungen des Ich-Erlebens oder Minus-Symptomatik liegen nicht vor. Ebenfalls existieren kaum Untersuchungen zum Langzeitverlauf von Symptomen und Therapieeffekten oder zu möglichen Residualsymptomen, wie sie bei schizophrenen Psychosen beschrieben werden, was bei einer postulierten gemeinsamen Ätiologie von Psychose und Demenz therapeutisch relevant wäre. Wegen der Eskalation der Symptomatik wäre es wünschenswert, ein praktikables Instrument für die frühe Diagnose einer Psychose bei Patienten mit Morbus Parkinson zur Hand zu haben, wie es kürzlich von Mitarbeitern der Klinik der Neurologie der Universität Marburg vorgestellt wurde (2).

\section{Psychosis in patients with Parkin- son's disease: Etiology, symptoms and treament}

Psychosis occurs in 10 to $40 \%$ of patients with Parkinson's disease. As a first measure, possible infectious, toxic, or metabolic causes, in particular hydration and electrolytes should be corrected if necessary. Anti-Parkinsonian medication should be reduced or even discontinued including anticholinergics, selegeline, dopamineagonists, and COMT inhibitors. Levodopa dosage should be reduced as much as possible. However, withdrawal of Antiparkinsonian medication usually worsens motor symptoms and is badly tolerated. Therefore, neuroleptic treatment will be often necessary and certain newer neuroleptics can be used because of their lower potency to induce or worsen extrapyramidal motor symptoms. Risperidone and olanzapine appear to cause deterioration of motor functions. Most data, also from controlled studies, show good efficacy of clozapine. However, side effects including sedation, orthostatic hypotension, sialorrhoea, and its potential to cause agranulocytosis, may be limiting. Adverse effects of quetiapine are sedation and orthostatic hypotension, however, case reports and caseseries in more than 200 Parkinson patients suggest that quetiapine is effective and well tolerated. Ziprasidone showed good antipsychotic effects and little side effects in case reports, also when the drug was administered by intramuscular application for agitated behavior. There are no data about efficacy and adverse effect profile of amisulpride and aripiprazole even though their profile shown in patients with schizophrenic psychosis suggests good efficacy and tolerability.

\section{Key words}

delusions - hallucinations -

Parkinson's disease - psychosis neuroleptics - dementia

\section{Literatur}

1. Braak H, Rub U, Braak E. Neuroanatomie des Morbus Parkinson. Veränderungen des neuronalen Zytoskeletts in nur wenigen für den Krankheitsprozess empfänglichen Nervenzellen führen zur progredienten Zerstörung umschriebener Bereiche des limbischen Systems. Nervenarzt 2000; 71: 459469

2. Brandstädter D, Spieker S, Ulm G, Siebert U, Eichhorn TE, Krieg JC, Ortel WH, Eggert K. Development and evaluation of the Parkinson Psychosis Questionnaire: A screening-instrument for the early diagnosis of drug-induced psychosis in Parkinson's disease. J Neurol 2005; epub ahead of print

3. Fernandez HH, Trieschmann ME, Friedmann JH. Treatment of psychosis in Parkinson's disease: safety, considerations. Drug Safety 2003; 26: 643-659

4. Goetz CG, Curgian LM, Leugans S. Hallucinations and sleep disorders in PD: six-year prospective longitudinal study. Neurology 2005; 64: 81-86

5. Gome-Esteban JC, Zarranz J], Velasco F, Lezcano E, Lachen MC, Rouco I, Barcena J, Boyero S, Ciordia R, Allue I. Use of ziprasidone in parkinsonian patients with psychosis. Clin Neuropharmacol. 2005; 28: $111-$ 114

6. Lemke MR. Clozapin: Rechtliche Aspekte der Verschreibung. Psychopharmakotherapie 1997; 4: 42

7. Lemke MR. Depression und Morbus Parkinson: Klinik, Diagnose, Therapie. Uni-Med Verlag, Bremen, Boston, 2002

8. Lemke MR. Effect of Reboxetine on Depression in Parkinson's Disease. J Clin Psychiatry 2002; 63: 300-304

9. Lemke MR. Neuropsychiatric Aspects of Parkinson's Disease. Focus on Parkinson's Disease 2004; 16: A44-48

10. Oechsner M, Korchounov A. Parenteral ziprasidone: a new atypical neuroleptic for emergency treatment of psychosis in Parkinson's disease? Hum Psychopharmacol. 2005; 20: 203-205

11. Papapetropoulos S, Mash DC. Psychotic symptoms in Parkinson's disease. From description to etiology. J Neurol 2005; 252: 753-764

12. Poewe W, Seppi K. Treatment options for depression and psychosis. J Neurol 2001; 248 S3: III12-21

13. Weiden P, Iqbal N, Mendelowitz A, Tandon R, Zimbroff D, Ross R. Best clinical practice with ziprasidone: update after one year of experience. J Psychiat Practice 2002; 8: 81-98

14. Witt K, Reiff J, Deuschl G. Die Therapie medikamenteninduzierter psychotischer Symptome bei Morbus Parkinson. Aktuelle Neurologie 2004; 31: 361-365

15. Wolters EC, Berendse E. Management of psychosis in Parkinson's disease. Curr Opin Neurol 2001; 14: 499-504

\section{Korrespondenzadresse:}

Priv.-Doz. Dr. med. Matthias R. Lemke

Ärztlicher Direktor

Rheinische Kliniken Bonn

Kaiser-Karl-Ring 20

53111 Bonn

mr.lemke@lvr.de

www.rk-bonn.lvr.de 